\title{
Emprendimientos y modelos matemáticos de Transporte y logística en la ciudad de Riobamba
}

\section{Freight and mathematical models and logistics transport enterprises in the city of Riobamba}

Susana del Rocío Guerrero Godoy. ${ }^{1}$, Jenny Margoth Villamarin Padilla. ${ }^{2}$ \& Monserrath Amparo Padilla Muñoz. ${ }^{3}$

\begin{abstract}
.
DOI: https://doi.org/10.33262/concienciadigital.v3i3.1318

Transport is considered of great importance at the international level has allowed great positive impacts that allows large-scale economic evolution, even more so in developing countries in small cities, the undertaking of transport in recent years is represented in the influx of heavy transport operators, light executives, urban that in one way or another impacts the local economy. In this study, you determine the number of operators registered in our city, as it is considered a small city but there are many vehicles destined to generate local and interprovincial income, which boost economic activity in the country, the logistics infrastructure that our The city still has some pending in terms of planning added to this the vehicular income of other provinces.
\end{abstract}

Keywords: Logistics. entrepreneurship, organization, transport, market, globalization.

\section{Resumen.}

El transporte es considerado de gran importancia a nivel internacional ha permitido grandes impactos positivos que permite la evolución económica a gran escala, más aún en países considerados en vías de desarrollo en pequeñas ciudades el emprendimiento del

\footnotetext{
${ }^{1}$ Escuela Superior Politécnica del Chimborazo, Facultad de Administración de Empresas, Unidad de Nivelación y Admisión, Riobamba, Ecuador, susana.guerrero@espoch.edu.ec

2 Escuela Superior Politécnica del Chimborazo, Facultad de Administración de Empresas Escuela Gestión de Transporte, Riobamba, Ecuador, jenny.villamarin@espoch.edu.com

${ }^{3}$ Escuela Superior Politécnica del Chimborazo, Facultad de Administración de Empresas Escuela Gestión de Transporte, Riobamba, Ecuador, monserrath.padilla@espoch.edu.com
} 
transporte en los últimos años se ve representado en la afluencia de operadoras de transporte pesado, livianos ejecutivos, urbano que de una forma u otra impacta en la economía local. En el presente estudio determinas la cantidad de operadoras registradas en nuestra ciudad, pues es considerada una ciudad pequeña, pero existe gran cantidad de vehículos destinados a generar ingresos locales, e interprovinciales los que dinamizan la actividad económica en el país, la infraestructura logística que cuenta nuestra ciudad aún tiene algunos pendientes en cuanto a planificación sumado a esto el ingreso vehicular de otras provincias.

Palabras clave: Logística, emprendimiento, organizaciones, transporte; marketing.

\section{Introducción.}

La movilidad de carga forma, sin duda, una columna fundamental en la tendencia económico industrial de cualquier país. logística que permite dinamizar el flujo de los productos, y en la cual se encuentran sumidos cerca del $45 \%$ al $50 \%$ de los costos logísticos totales de una compañía, lo que la convierte en un factor clave del éxito para la entrega oportuna de materia prima y productos acabados a los clientes finales de la cadena logística, generador de valor para la organización.

El transporte de carga tradicional y la distribución se ha enfocado en el transporte físico de mercancías, donde la diferenciación ha sido el flete (Mora, 2015)

En esta ciudad el ingreso de vehículos por el peaje San Andrés fue de 12.548 vehículos de todo tipo, su infraestructura logística presente en los centros de acopios en donde el transporte de carga tradicional y distribución es normal en los mercados uno de los más importantes es el mercado mayorista de la ciudad que solo para el año 2018 ingresaron 1.030.107 vehículos y cuenta con un catastro de comerciantes aproximadamente de 948 miembros y 183 arrendatarios temporales de diferentes productos provenientes de los cantones de la provincia y sus destinos principales ciudades de la Costa y del Oriente.

Al hablar de transporte se fundamenta la palabra responsabilidad social Corporativa que mantienen un equilibrio en un modelo de desarrollo eficaz aún menor costo posible y el respeto al medio ambiente asume y resume los costos sociales dentro de las empresas.

\section{Planteamiento del problema.}

El incremento de vehículos en la ciudad de origen interno y externo necesitan una infraestructura logística moderna y eficiente en nuestra ciudad.

\section{Justificación de la investigación.}

El comercio sin duda es productor de economía local y nacional la construcción logística presente y creadas para este fin no persistentemente no son las más adecuadas peor aun cuando justificamos el incremento de vehiculas cada año como es el caso específico del 
mercado mayorista el año 2017 fue de 976,301 mientras que en el 20181.030107 vehículos a esto adicional el ingreso de coches de otras provincias.

Las cooperativas de carga liviana son las siguientes
1. 5 de junio,
2. Condorazo
3. El camal
4. Juan de Velasco
5. Julio Román
6. Mariscal sucre
7. San Cristóbal
8. Unión y fuerza

Analizar los incrementos de los emprendimientos en la ciudad de Riobamba y evolución de las Pymes.

\section{Marco teórico.}

Ecuador es uno de los países más individuos dispuestos a emprender de América Latina. según lo señala el artículo Global Entrepreneurship Monitor GEM Ecuador 2013, expuesto en las ciudades de Quito y Guayaquil, y conocido por ser uno los censores de mayor emprendimiento. El índice de labor emprendedora anticipada por individuos de menores de edad o TEA se situó en el 2013 en el 36\%.

el año pasado "cerca de 1 de cada 3 adultos ecuatorianos había comenzado los trámites para fundar un negocio, o tenía uno cuya experiencia no superaba los tres años y medio", según el artículo. El dato del GEM 2013, conjuntamente, confirma que existe un incremento continuo de la actividad emprendedora temprana.

En el 2009, el índice fue de 15,8\% y en el 2012 llegó a 26,6\%. Sin confiscación, existe un dato que inquieta: la extensión del emprendimiento por escasez o necesidad; es decir, por falta de otras opciones de entrada.

Según el estudio, el 22,7\% de los adultos aseveró haber iniciado aprovechando una oportunidad en el mercado, sin embargo, que el 12,1\% lo hizo por escasez de fuentes de trabajo. Con esas cifras, Ecuador saltó a tener la tasa de emprendimientos por necesidad más alta del territorio, continuo de Perú $(5,25 \%)$ y Brasil (4,95\%).

Según investigadores consultados, el emprendimiento por necesidad está emparentado con los índices de personas sin trabajo (5,58\% hasta marzo) y pequeños emprendimientos (44,25\% hasta marzo). las cifras, que pueden trascender alejados y frías, el compatriota que emprende está reflexivo de las dificultades que existen para articular y sostener un negocio. 


\section{Emprendedor.}

Emprendedor es un vocablo que tiene muchos axiomas, de acuerdo al ámbito en el que se localice.

- En las actividades comerciales Emprendedor es sinónimo de Empresario (dueño de una empresa con finales de lucro, es decir persigue un beneficio.

- Académico un alumno igualmente es un emprendedor.

En si es la capacidad de dar uso a sus recursos disponibles, identificando a personas capaces de identificar oportunidades y y elaborar un nuevo concepto de negocio.

Definición. Emprendedor persona con autoconfianza y características que le permiten ver oportunidades y generar conceptos de negocio a partir de la creación, la innovación o el mejor empleo de los recursos (Alcaraz, 2011)

\section{Visión histórica del término empresario.}

El concepto de empresario ha sufrido grandes modificaciones desde la época del capitalismo mercantilista hasta la actualidad. De ser el propietario de la empresa y el que tomaba las decisiones, ha pasado a constituirse en una figura mucho más compleja, que no necesariamente tiene que ser una persona física ni el dueño de la empresa.

\section{Resumen.}

Siglos XVIII-XIX el mercader sedentario

Siglo XIX: el empresario organizador

Siglo XX:

- Empresario como persona que asume un riesgo

- Empresario como persona innovadora

- El empresario en la actualidad

Siglos XVIII-XIX: el mercader sedentario (Suerte en su negocio) (Salas Salazar. Inca Falconi, 2019)

Actualmente el administrador ha de ser transformador, al mismo tiempo de un buen líder y saber ser un buen táctico para escoger los vías adecuados para conseguir los objetivos.

La nueva imagen del empresario cumple las siguientes características: 
- El director ya no es irreparablemente el propietario de la empresa, aunque en las compañías de pequeño tamaño siga existiendo así. Esto se debe a la separación entre la propiedad y el control de esta.

- El director no tiene por qué ser una única persona. En varios casos, sobre todo en las compañías de mayor tamaño, el empresario se transforma en un órgano colegiado, como sucede con el consejo de administración de las sociedades anónimas.

- La actividad del director no tiene por qué ser de tipo perito. Más bien se dedica a establecer objetivos, establecer planes, organizar la empresa, etc. Esta función se denomina administración, o management.

- La Responsabilidad Social Empresarial (RSE) es el compromiso continuo de la empresa de contribuir al desarrollo económico sostenible, mejorando la calidad de vida de sus empleados y sus familias, así como la de la comunidad local y de la sociedad en general [1]. Ser Socialmente Responsable significa ser consciente del efecto que nuestros actos pueden ocasionar. Implica una gestión estratégica que se planifica, ejecuta y mide, como toda gestión del negocio, debe ser transversal a la empresa, ya que implica todos los niveles de esta y no un área específica. No hay empresa socialmente responsable si sus empleados y sus líderes no lo son. La RSE no es un costo, es una inversión de retorno a mediano y largo plazo (ANTELO-GONZÁLEZ, 2015)

Superando a pise como Colombia, Perú y Chile lo cual es bueno para la economía ecuatoriana actual

La situación del emprendimiento analizada inclusive con un estudio realizado por el autor en el año 2016 impulsa a proponer un modelo de desarrollo del emprendimiento bajo las teorías de gestión y administración de negocios.

Gestión del Emprendimiento comprendiéndose como las acciones o diligencias a realizarse para hacer posible una buena empresa, considera que el aporte técnico no solo se debería encaminar a la creación del negocio sino al desarrollo del mismo para convertirse en una organización competitiva con la capacidad suficiente de ofertar empleos e ingresos representativos a la sociedad ecuatoriana

(Quishpe, 2018)

- Entrepernar. - Es la persona que aplica la conducta emprendedora en la creación y manejo de sociedades propias.

- Intrapreneur. - Quien lo aplica dentro de empresas que no son suyas.

Alcanzamos a la terminación que existen múltiples significados o definiciones 


\section{Características del emprendedor.}

Un emprendedor es un individuo de negocios que no sólo concibe y organiza empresas, sino que continuamente toma peligros al hacerlo. No todos los profesionales autónomos son verdaderos individuos emprendedores, y no todos los emprendedores se opinen de la misma forma. Sin embargo, existen algunos tipos comunes entre ellos:

\section{Definición de señal de seguridad.}

Es un objeto físico que, sirviéndose de la combinación de una forma geométrica, un color y un símbolo proporciona una información determinada relacionada con la seguridad.

\section{Clases de señales de seguridad.}

En función de su aplicación se dividen en:

- Señales de prohibición: Señal de seguridad que impide una actuación que puede provocar un contexto de peligro.

- Señales de obligación: Es una señal de seguridad que exige a una conducta determinado.

- Señales de advertencia: Señal de seguridad que indica un peligro.

- Señales de información: Señal que facilita información para facilitar el socorro o garantizar la seguridad de las personas.

- Señal de salvamento: Es la señal que en caso de riesgo indica la salida de ocurrencia, la situación del lugar de socorro o la colocación de un dispositivo de salvamento.

- Señal indicativa: Suministra otras investigaciones distintas a las de prohibición, contrato y de aviso.

- Señal auxiliar: Contienen exclusivamente texto y se utiliza conjuntamente con las señales indicadas anteriormente.

- Señal adicional de riesgo permanente: Sirven para señalar lugares donde no se utilicen formas precisas reguladas y que suponen un riesgo constante de choque, caída.

\section{Colores, formas, dimensiones y símbolos.}

Colores: Su ocupación es llamar la atención, mostrar la existencia de un riesgo y facilitar su identificación fácil y rápidamente. 
Las zonas de los signos donde se aplica el color son tres:

- Zona de seguridad.

- Zona de contraste.

- Zona de símbolo.

\section{Señalización horizontal.}

\section{Definición.}

La señalización horizontal, corresponde a la aplicación de estampillas viales, conformadas por líneas, flechas, insignias y letras que se pintan sobre el suelo, bordillos o sardineles y colocaciones de las vías de circulación o adyacentes a ellas, así como los sustancias que se colocan sobre la superficie de rodadura, con el fin de normalizar, canalizar el tránsito o indicar la apariencia de obstáculos.

\section{Consideraciones generales.}

La demarcación rescata ocupaciones definidas e significativos en un adecuado esquema de ordenación del tránsito. En algunas cuestiones, son usadas para terminar las órdenes o avisos de otros terminales, tales como las señales verticales y semáforos; en otros, comunican instrucciones que no pueden ser mostradas mediante el uso de ningún otro conector, siendo un modo muy cierto de hacerlas entendibles.

Para que la señal horizontal efectúe la función para la cual se usa, se solicita que se tenga una igualdad respecto a las extensiones, diseño, símbolos, caracteres, colores, periodicidad de uso, condiciones en que se emplea y tipo de material usado.

Las marcas viales o demarcaciones deben ser reflectivas excepto paso peatonal tipo cebra, o estar debidamente iluminadas.

Las líneas de demarcación con pintura en frío que se empleen sobre concreto asfáltico deberán ser pintadas como mínimo treinta (30) días después de edificada la carpeta de rodadura. Cuando por argumentos especiales se solicite realizar el territorio antes de dicho término, ésta deberá realizarse empleando un grosor húmedo igual al medio del especificado para el apunte definitivo y se deberá colocar dentro de los ocho (8) días siguientes.

\section{Materiales:}

Las estampillas viales deben hacerse acorde al uso de pinturas en frío o en caliente. pero puede utilizarse nuevo tipo de material, siempre que cumpla con los detalles de color y visibilidad; siendo preciso que no muestren condiciones resbalosas, esencialmente en los pasos peatonales y en los entornos a éstos. 
Para completar las líneas alargados, podrán utilizarse unidades individuales (tachas, estoperoles o pintura termoplástica con pequeños abultamientos-viralice), que sobresalgan menos de $2,5 \mathrm{~cm}$ de la superficie del pavimento y de color blanco o amarillo.

Para demarcar cabos o islas, podrán monopolizar otras unidades (tachones, boyas metálica o plásticas, bordillos, etc.), que sobresalgan de la superficie del pavimento a una altura máxima de $10 \mathrm{~cm}$.

Las obligaciones que debe efectuar la pintura en frío para demarcación de suelos son los vistos en la norma técnica colombiana NTC-1360-1. En el caso de las tachas reflectivas corresponderá efectuar con lo desarrollado en la norma técnica colombiana NTC-4745.

\section{Pasos para seguir en la tramitación de problemas}

\section{Investigación.}

Lo más normal es que, al principio, los problemas no tengan una representación claramente definida. Para igualar debemos crear preguntas de este tipo:

- ¿Cuáles dificultades debemos resolver?

- ¿Por qué creemos que no estamos en el nivel de desempeño apropiado?

- ¿Cuáles son nuestras fines explícitas e implícitas?

Pensamiento disidente.

Pensamiento fusionado.

Exploración Igualar problemas y oportunidades.

Concentrarse en el fin Búsqueda de encuesta.

Coleccionar datos y puntos de vista.

Identificar la información más relevante.

Personalización del problema.

Edificar diferentes problemas posibles Elegir el problema más prometedor.

Investigación de soluciones.

Desplegar muchos dilemas para el medio para Elegir las ideas más viables Evaluación de soluciones. 
Fundar diferentes criterios para valorar los medios Igualar los criterios más apropiados Ejecución de un medio Nivelar varios elementos que accedan implementar la solución

Llevar a cabo la solución más prometedora.

\section{Metodología de la investigación.}

\section{Tipo de investigación.}

El análisis es el método empírico de estudio de un objeto, en el cual el investigador crea las situaciones necesarias o adecua las existentes, para la aclaración de las propiedades y relaciones del objeto, que son de utilidad en la investigación

\section{Tipo de estudio.}

Se aplicará un estudio Transversal, debido a que no existe continuidad en el eje del tiempo, es decir que se mide a la vez la prevalencia de la exposición y del efecto en una muestra poblacional en un solo momento temporal.

\section{Métodos de investigación.}

Método Inductivo: Este procedimiento parte de lo personal a lo general, ya que inicia con la información de los problemas individuales de derivadas del emprendimiento en el transporte como fuente de trabajo para poder llegar a conclusiones generales sobre la realidad de esta.

Método Analítico: a través de este método se creerá cada efecto obtenido para detectar las posibles causas que ocasionan falencias.

\section{Técnicas de la investigación.}

Las técnicas practicadas para la cosecha de información son:

- Información secundaria: Para la presente investigación se pretende recolectar información de entes gubernamental, la misma que permiten estar al tanto las opiniones, actitudes e indicaciones.

\section{Observación directa.}

Para el progreso de este proyecto el equipo de investigación obtiene investigación directa en las principales vías de la ciudad

\section{Instrumentos.}

Cuestionario: La aplicación de una encuesta con preguntas cerradas y abiertas dirigidas a los usuarios de las vías determinando sus expectativas y percepción. 
Ficha de observación: Se utiliza un check list para tiempos, calidad y duración del objeto de estudio junto con la calidad de las vías.

\section{Alcance del proyecto.}

Este estudio tiene como alcance el análisis del incremento vehicular en la ciudad de Riobamba analizando la información proporcionada por varias instituciones gubernamentales

\section{Desarrollo de la investigación.}

Nuestra investigación se basará en los siguientes parámetros:

\section{Métodos.}

\section{Científico.}

Caracterizado por tener un grupo de pasos para realizar un experimento y de ahí obtener una respuesta o conclusión del mismo.

\section{Inductivo.}

Método que obtiene conclusiones generales a partir de premisas particulares; lo que se debe analizar los aspectos generales tanto variables dependientes e independientes.

\section{Deductivo.}

Requiere de principios generales, conocimientos bastos sobre el tema para llegar a cualquier conclusión de orden particular. Lo que en la investigación mediante un razonamiento lógico deducirá por qué mejorar el servicio de calidad y el funcionamiento del sistema operacional del terminal.

\section{Analítico.}

Este método implica el análisis es decir la separación de un todo en sus partes o elementos constitutivos. Lo que permitirá hacer el análisis del sistema operacional del terminal Inter cantonal para lograr una mejor funcionalidad al mismo.

\section{Sintético.}

Es un proceso analítico - sintético mediante el cual se parte del estudio de casos, hechos o fenómenos particulares para llegar al descubrimiento de un principio o ley que rige. Es decir, en nuestro proyecto de investigación es la estructuración y agrupación de datos para desarrollar las conclusiones respectivas.

\section{Técnicas.}




\section{Observación directa.}

Es la observación directa y análisis de la información tipo secundaria obtenida

\section{Resultados.}

\section{Análisis e interpretación de resultados.}

La información obtenida con los diferentes actores del transporte evidencia que esta actividad para muchos es una forma de emprendimiento comienza con cooperativas de transporte liviana la cual va en incremento paulatino como arroja la siguiente información.

Tabla 1. Información acerca del tráfico promedio anual (TPDA) del peaje de San Andrés del año 2018 por categorías, ubicado en la Provincia de Chimborazo.

\begin{tabular}{lccc}
\hline & \multicolumn{3}{c}{ PEAJE SAN ANDRÉS } \\
CATEGORIA & AÑ & \multicolumn{1}{c}{ MES } & TPDA \\
\hline Livianos & 2018 & enero - diciembre & 9.413 \\
Camión ejes & 2018 & enero - diciembre & 889 \\
Buses 2 ejes & 2018 & enero - diciembre & 8 \\
Camión 3 ejes & 2018 & enero - diciembre & 1309 \\
Buses 3 ejes & 2018 & enero - diciembre & 188 \\
Camión 4ejes & 2018 & enero - diciembre & 26 \\
Camión 5 ejes & 2018 & enero - diciembre & 68 \\
Motos & 2018 & enero - diciembre & 76 \\
Exonerados & 2018 & enero - diciembre & 114 (vehículos, 2018) \\
& & TOTAL 12.245 & \\
\hline
\end{tabular}

Fuente: MTOP (MTOP, 2018)

Gráfico 1. Información acerca del tráfico promedio anual (TPDA) del peaje de San Andrés del año 2018 por categorías, ubicado en la Provincia de Chimborazo.

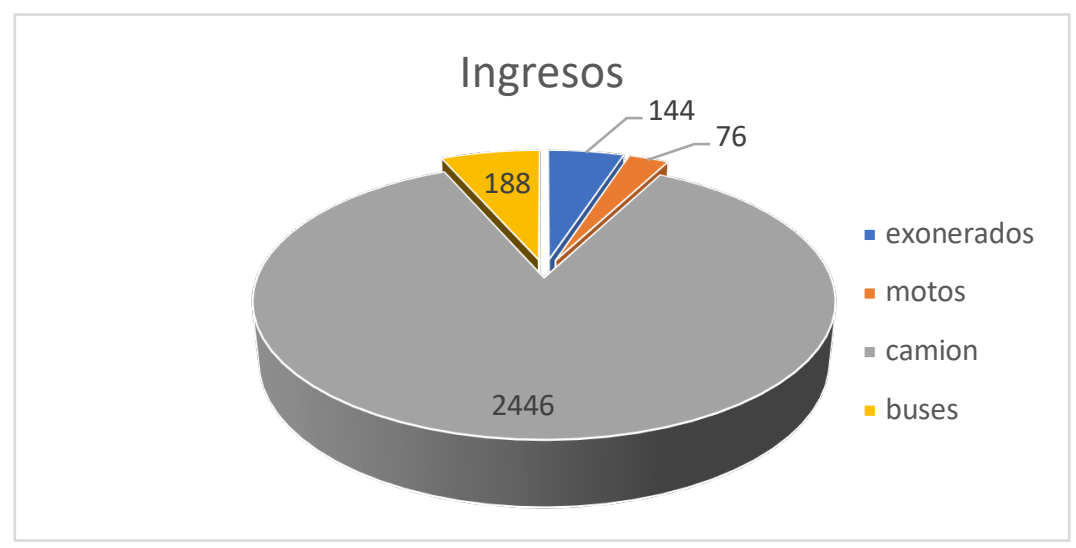

Fuente: MTOP (MTOP, 2018) 
Análisis. El peaje de san Andrés demuestra solo en el mes de enero la gran cantidad de camiones que ingresas a nuestra ciudad representada con el color gris.

Tabla 2. Inventario de cooperativas interprovinciales de transporte en Riobamba

\begin{tabular}{ll}
\hline Inventario de cooperativas interprovinciales de transporte en Riobamba \\
\hline Interprovincial Riobamba & $\begin{array}{l}\text { Compañía de transporte interprovincial } \\
\text { Condorazo 11unidades } \\
\text { Cooperativa de transporte de pasajeros en buses } \\
\text { "Trans vencedores" 20 unidades }\end{array}$ \\
Interprovincial Riobamba & $\begin{array}{l}\text { Cooperativa de transporte interprovincial de } \\
\text { pasajeros en buses unidos } 41 \text { unidades }\end{array}$ \\
Interprovincial Riobambrovincial Riobamba & $\begin{array}{l}\text { Cooperativa de transporte interprovincial Patria } \\
\text { Interproperativa de transporte interprovincial san }\end{array}$ \\
Interprovincial Riobamba & $\begin{array}{l}\text { Cooper de Ilapo } \\
\text { Cooperativa de transporte Riobamba }\end{array}$ \\
Interprovincial Riobamba & $\begin{array}{l}\text { Cooperativa de transportes de pasajeros Ecuador } \\
\text { ejecutivo } \\
\text { Cooperativa de transporte público de pasajeros } \\
\text { interprovincial Sangay } \\
\text { Interprovincial Riobamba }\end{array}$ \\
Interprovincial Riobamba & $\begin{array}{l}\text { Cooperativa de transportes interprovincial } \\
\text { Chimborazo }\end{array}$ \\
Interprovincial Riobamba & $\begin{array}{l}\text { Cooperativa de transportes Nuca Llacta } \\
\text { Cooperativa interprovincial de pasajeros en buses } \\
\text { Colta }\end{array}$ \\
\hline Interprovincial Riobamba
\end{tabular}

Fuente: ANT (ANT, Cooperativas de transporte, 2018)

Según la información Presentada por ANT son 11 las cooperativas presentes en esta Ciudad sin tomar en cuenta las cooperativas de carga pesada que es otra actividad de las empresas de servicios de transporte.

Tabla 3. Operadoras interprovinciales

\begin{tabular}{lc}
\hline \multicolumn{1}{c}{ Operadoras } & Canton \\
\hline Cooperativa de transporte interprovincial de pasajeros cta. & Alausi \\
Cooperativa de transporte de pasajeros de buses Chunchi & Chunchi \\
Cooperativa de transportes Guamote & Guamote \\
Cooperativa de transportes Andina & Guano \\
Cooperativa de transporte 20 de diciembre & Guano \\
Compañía de transportes Condorazo cia. Ltda. & Riobamba \\
Cooperativa de transporte de pasajeros en buses Trans Vencedores & Riobamba \\
Cooperativa de transporte interprovincial de pasajeros en buses unidos & Riobamba \\
\hline
\end{tabular}


Cooperativa de transporte interprovincial Patria

Riobamba

Cooperativa de transporte interprovincial san lucas de Ilapo

Riobamba

Cooperativa de transporte Riobamba

Riobamba

Cooperativa de transportes de pasajeros Ecuador ejecutivo

Riobamba

Cooperativa de transportes de pasajeros Sangay

Riobamba

Cooperativa de transportes interprovincial Chimborazo

Riobamba

Cooperativa de transportes Nuca Llacta

Riobamba

Cooperativa interprovincial de pasajeros en buses Colta

Riobamba

Riobamba Express cia. Ltda.

Riobamba

Fuente: ANT (ANT, 2018)

Estas cooperativas incluyen las siguientes resoluciones.

\section{Resoluciones.}

- Constitución jurídica

- Permiso de operación

- Alcance del permiso de operación

- Contrato de operación

- renovación de permiso de operación

- Rectificación de renovación del permiso de operación

- Renovación de rutas y frecuencias

- Incremento de cupo

- Cambio de vehículo

- Cambio de socio/vehículo

- Des habilitación de vehículo

- Cambio de socio

- Cambio de socio/habilitación de vehículo

- habilitación de vehículo

- Rectificaciones de las resoluciones

- Concesión de rutas y frecuencias 
- Modificación de rutas y frecuencias

- Reforma de estatuto

- Adenda al contrato de operación

- Adenda de renovación de permiso de operación

- Adenda de incremento de cupo

- Adenda habilitación de vehículo por incremento de cupo

- Adenda de cambio de vehículo

- Adenda de cambio de socio

- Adenda de cambio de socio / vehículo

- Adenda de des habilitación de vehículo

- Adenda de cambio de socio / habilitación de vehículo

- Adenda de habilitación de vehículo

- Adenda de rectificaciones de las resoluciones

- Adenda de reforma de estatuto

Tabla 4. Operadoras de transporte escolar EINST

\begin{tabular}{lcl}
\hline Cooperativa & Socios & Nombre representante \\
\hline Corazon de la patria & 63 socios & Sr Angel Haro \\
Tranescordan & 17 cupos & Sr Alonso Mereano \\
Padremensi & 14 socios & Sr Manuel Inca Sifuentes \\
Riobusetas & 25 socios & Sr Ramon Martinez \\
Sanpertrans & 8 socios & Sr Cornelio Tello \\
Total & 127 socios & \\
\hline
\end{tabular}

Fuente: ANT (ANT, 2018)

Elaboración: Equipo de Investigación. 
Tabla 5. Operadoras de taxis ejecutivos

\section{OPERADORAS DE TAXIS EJECUTIVOS}

\begin{tabular}{|c|c|c|c|c|}
\hline Cooperativa & Socios & $\begin{array}{l}\text { Nombre represt. } \\
\text { Legal(presidente) }\end{array}$ & Dirección sede & Telf. \\
\hline El amanecer & 17 socios & Carlos Naranjo & Perimetro de las industrias Canchon & 22374494 \\
\hline $\begin{array}{l}\text { Frenaten } \\
\text { Chimborazo }\end{array}$ & 45 socios & Luis Cajas & Calle principal barrio el Carmen & $2300-065$ \\
\hline Prestomovil & 26 socios & Julio Aucancela & Junin entre Alvarado y Quitumbe & 2960750 \\
\hline Taxamanecer s.a & 18 socios & Alfonso Machado & $\mathrm{Km} 2$ 1/2 via a baños sector Lacas & 2372200 \\
\hline Vallshyris s.a & 21 socios & Tene Lobato Diego Armando & $\begin{array}{l}\text { Padre Lobato y Garcia Moreno } \\
\text { Yaruquies }\end{array}$ & $2618-082$ \\
\hline Predretaxi s.a & 30 socios & Luis Muñoz & $\begin{array}{l}\text { Av Atahualca barrio el Pedregal via } \\
\text { Santa Cruz }\end{array}$ & $2614-440$ \\
\hline $\begin{array}{l}\text { Primicia de los } \\
\text { andes }\end{array}$ & 24 socios & Jorge Perez & Barrio el Batan & 32222127 \\
\hline Taxmovilence & 18 socios & Carlos Muñoz & $\begin{array}{l}\text { Av. Juan Felix Proaño e Irlanda } \\
\text { barrio la Libertad }\end{array}$ & $2300-583$ \\
\hline Translaureles & 27 socios & Alfredo Jiron & Av. Ciurcunvalación y puruha & 987041893 \\
\hline Santanitanor & 26 socios & Sr anibal Sanchez & Coope de vivienda Santa Anita norte & 983488993 \\
\hline Elmadrigal & 15 socios & Vinicio Cordova & San Jose de Chibunga & 2962153 \\
\hline Montecarlo & 10 socios & Eduardo Davalos & Nuevas & 2612529 \\
\hline Ejecutrans del río & 15 socios & Paredes Perez Rosa Georgina & Av. Daniel 1 Borja & 2600619 \\
\hline Riobambeñita & 22 socios & Sra Martha Guarquila & Segundo Rosero y Miguel Jijon & 982661469 \\
\hline Cisneros trans & 15 socios & $\begin{array}{l}\text { Carrillo Velastegui Angel } \\
\text { Guillermo }\end{array}$ & Bulgaria y Rusia & 2370731 \\
\hline Los evac losvack & 15 socios & Edgar German Mejia Armas & $\begin{array}{l}\text { Av. Monseñor } 1 \text { Proaño y Cesar } \\
\text { Dávila (pasaje los Andes) }\end{array}$ & 2300200 \\
\hline Ciclontaxi & 15 socios & Estalin Patricio Pilco Taco & Km1 1/2 via a Baños & \\
\hline Lubuvir & 15 socios & Kleber Chavez & $\begin{array}{l}\text { Juan de Dios Martínez y Diego } \\
\text { Rivera }\end{array}$ & \\
\hline Ejecusevice & 15 Socios & $\begin{array}{l}\text { Padilla Sampedro David Mesias } \\
\text { Cdla. } 24 \text { de Mayo la }\end{array}$ & $\begin{array}{l}\text { Panamericana norte barrio Altamiras } \\
\text { del norte }\end{array}$ & \\
\hline
\end{tabular}

Fuente: ANT (ANT, 2018)

Gráfico 2. Ventas

Ventas

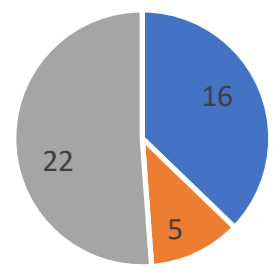

- opperadoras interprovincial oeradoras de transporte escolar

- oeradoras de taxis ejecutivos

Fuente: ANT (ANT, 2018) 
Tabla 6. Resumen

\begin{tabular}{lc}
\hline Cooperativas interprovinciales de & transporte en Riobamba \\
Operadoras de trasnporte escolar EINST & 16 \\
Operadoras de taxis Ejecutivos & 5 \\
\hline
\end{tabular}

Fuente: Elaboración propia.

\section{Análisis.}

La grafica demuestra que existen mayor gran cantidad de operadoras de taxis ejecutivos en nuestra ciudad superando a las otras operadoras de transporte.

\section{Análisis de la distribución del transporte pesado.}

Detallado el incremento de empresas de transporte que las podemos denominar emprendimientos en algunos de los casos el de mayor números son las operadora de taxis ejecutivos, el problema está en las cooperativas de carga pesada radical en su distribución en la que se determine los costó, y nivel de servicio ya que este es diverso por lo que tomamos diferentes ejemplos servicio de encomiendas

Sus prioridades:

- Costos, red, y centro de entrega

- Ingresos según 1 densidad de la red

En transportes de carga.

Se debería calcular:

- Costos de operación de la flota de transporte

- Perdidas de los vehículos por diferentes motivos, que nos permita obtener el total de costos

Las operadoras logísticas si agrandamos el caso 4pl más completas como ejemplo (Transporte, distribución, programación e inventarios) 4pl se crearía módulos específicos para calcular los costos de cada uno de sus mercados y determinar el aumento de la demanda de cada servicio sin olvidar a la competencia y mercado.

Esto en relación con el precio sin rebajar la calidad en el servicio, y alterar la mezcla de servicios otorgados.

Claro está que el modelo funciona de acuerdo con sus necesidades para obtener un crecimiento correcto y expectativas de los usuarios o consumidor final el análisis de la calidad en el servicio mediante una retroalimentación del proceso de sus servicios ayudar a elaborar un modelo más correcto con el fin de obtener mejores tiempos. Costos, lo que se refleja en el presupuesto

En el caso de las empresas de encomiendas su modelo creado en base a:

- El volumen en relación con los puntos de veta o recepción

- El volumen de la inversión

- Su utilidad proyectada por lo mínimo 5 años, derivados de (ingresos proyectados) 
En el caso del transporte de carga:

- La pérdida es una función lineal creciente del tiempo de transporte.

- La pérdida es una función no lineal creciente de la antigüedad de los vehículos.

- La pérdida es una función lineal decreciente del tamaño

- Función lineal, $y=m x+b$

- Retornos progresivamente más grandes, $y=A X \quad b,>1$

- Retornos progresivamente más pequeños, $y=A X \quad b,<1$

\section{Resultados.}

El estudio demuestra que esta ciudad el incremento de emprendimientos de transporte va en crecimiento en el interior de la ciudad existente gran cantidad de operadora de taxis ejecutivos, mientras que el peaje de San Andrés nos demuestra el paso de Camiones por nuestra ciudad, hay que tomar en cuenta considerando que la ciudad se encuentra en un punto central del país y las frecuencias de destino crecen constantemente, los emprendimientos de transporte siempre serán presente nueve la economía del país.

\section{Conclusiones:}

- El sector de los servicios de transporte, al observar el número de cooperativas interprovinciales de carga y Liviana vemos que existen un número considerable dentro de nuestra ciudad, algunas cooperativas estimadas rentables otras no según sus dueños, lo que no tiene coherencia pues si no fuese rentable porque adquieren nuevas unidades para emprende

- La infraestructura logística de nuestra provincia visualizada en las vías de ingreso a la ciudad está en buen estado esto gracias a los Peajes como el de San Andrés, dentro de la ciudad la planificación del transporte urbano no tiene una organización adecuada lo que puedo influir en resultados negativos pues este estudio demuestra que el emprendimiento de transporte crece a pesar de no tener un estudio que ubica su verdadera necesidad ara ser generado.

- Al hablar el aspecto económico este emprendimiento genera economía local pues dentro del transporte de carga pesada la mayoría de las cooperativas son utilizadas para llevar materiales y legumbres a las ciudades del oriente y costa.

- Si hablamos del transporte interprovincial verificamos que existen dueños de unidades que una cooperativa que también unidades en otras cooperativas asumiendo el conceto de rentabilidad.

\section{Referencias bibliográficas:}

Alcaraz. (2011). como emprender con éxito. MEXICO.

ANT. (2018). Operadoras de la provincia de Chimborazo. Riobamba.

ANT. (2018). Cooperativas de transporte. Cooperativas de Transporte. Riobamba. 
Antelo - González, A.-R. (2015). Responsabilidad social. Instituto Superior Politécnico José Antonio Echeverría Cuba.

Bibliógrafa (vehículos, 2018) (MarcadorDePosición1)fía

https://www.esumer.edu.co/images/centroeditorial/Libros/fei/libros/modelaciondesistem aslogisticos.pdf.

https://www.esumer.edu.co/images/centroeditorial/Libros/fei/libros/modelaciond esistemaslogisticos.pdf. Obtenido de https://www.esumer.edu.co/images/centroeditorial/Libros/fei/libros/modelaciond esistemaslogisticos.pdf.

Mora, L. (2015). logística y distribución de carga. En L. Mora, logística y distribución de carga. EcoEdiciones.

MTOP. (2018). ingreso y egreso de vehículos peje San Andrés 2018. ingreso vehicular. Riobamba.

Quishpe, M. L. (2018). situación y gestión del emprendimiento. ISBN: 978-9942-30-5800 Primera Edición (Ecuador): Enero del 2018 .

Salas Salazar. Inca Falconi, V. C. (2019). Gestión de emprendimientos y evolución de las pymes en el. digital.

Vehículos, M. i. (2018). Mtop ingreso de vehículos . Riobamba. 


\section{PARA CITAR EL ARTÍCULO INDEXADO.}

Guerrero Godoy, S. del R., Villamarin Padilla, J. M., \& Padilla Muñoz, M. A. (2020). Emprendimientos y modelos matemáticos de Transporte y logística en la ciudad de Riobamba. ConcienciaDigital, 3(3), 313-331. https://doi.org/10.33262/concienciadigital.v3i3.1318

\section{Ciencia}

El artículo que se publica es de exclusiva responsabilidad de los autores y no necesariamente reflejan el pensamiento de la Revista Conciencia Digital.

El artículo queda en propiedad de la revista y, por tanto, su publicación parcial y/o total en otro medio tiene que ser autorizado por el director de la Revista Conciencia Digital.
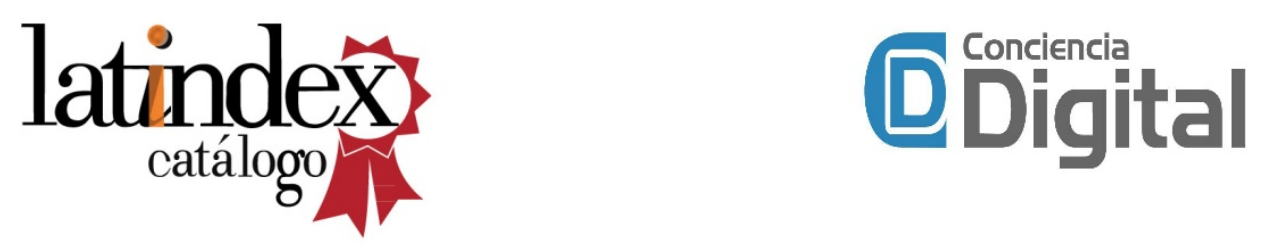